ARTICLE

\title{
Mammalian SRP receptor switches the Sec61 translocase from Sec62 to SRP-dependent translocation
}

Bhalchandra Jadhav ${ }^{1,2}$, Michael McKenna ${ }^{2}$, Nicholas Johnson ${ }^{2, \dagger}$, Stephen High ${ }^{2}$, Irmgard Sinning ${ }^{1}$

\& Martin R. Pool ${ }^{2}$

Two distinct pathways deliver secretory proteins to the Sec61 protein translocase in the endoplasmic reticulum membrane. The canonical pathway requires the signal recognition particle (SRP) and its cognate receptor (SR), and targets ribosome-associated proteins to the $\mathrm{Sec}$ translocase. The SRP-independent pathway requires the Sec translocase-associated ER membrane protein Sec62 and can be uncoupled from translation. Here we show that SR switches translocons to SRP-dependent translocation by displacing Sec62. This activity localizes to the charged linker region between the longin and GTPase domains of SR $\alpha$. Using truncation variants, crosslinking and translocation assays reveals two elements with distinct functions as follows: one rearranges the translocon, displacing Sec62 from Sec61. A second promotes ribosome binding and is conserved between all eukaryotes. These specific regions in $S R \alpha$ reprogramme the Sec translocon and facilitate recruitment of ribosome-nascent chain complexes. Overall, our study identifies an important function of SR, which mechanistically links two seemingly independent modes of translocation.

\footnotetext{
${ }^{1}$ BZH, University of Heidelberg, Im Neuenheimer Feld 328, D-69120 Heidelberg, Germany. ${ }^{2}$ Faculty of Life Sciences, University of Manchester, Michael Smith Building, Oxford Road, Manchester M13 9PT, UK. †Present address: Institute of Organic Chemistry and Biochemistry, Academy of Sciences of the Czech Republic, Flemingovo ńměstí 542/2, 16610 Prague 6, Czech Republic. Correspondence and requests for materials should be addressed to I.S. (email: irmi.sinning@bzh.uni-heidelberg.de) or to M.P.

(email: martin.r.pool@manchester.ac.uk)
} 
P roteins destined for secretion from the cell typically possess an N-terminal hydrophobic signal sequence, which targets them to the protein-translocating channel at the endoplasmic reticulum (ER) membrane ${ }^{1}$. Once the protein has been translocated across the membrane, the signal sequence is usually cleaved off and the mature protein can then fold and move through the secretory pathway via vesicular trafficking. Several distinct targeting pathways deliver proteins to the proteinconducting channel, which is formed by the heterotrimeric Sec61 complex $(\operatorname{Sec} 61 \alpha \beta \gamma)^{2}$. Perhaps the best-characterized pathway is the canonical signal recognition particle (SRP) pathway, which is conserved in all domains of life $e^{3,4}$. SRP is a ribonucleoprotein complex, which binds to ribosomes and can recognize the signal sequence as it emerges from the ribosome exit tunnel ${ }^{5,6}$. Recognition of the signal sequence by the SRP54 component of SRP leads to a transient retardation in translation concomitant with targeting to the ER membrane via an interaction with the cognate SRP receptor $(S R)^{7-10}$. SR mediates the transfer of the ribosome, together with the nascent chain, to the Sec61 translocase ${ }^{11}$. Translation resumes and the nascent chain is fed directly from the ribosome into the pore of the Sec61 translocon, which is co-aligned with the ribosome exit tunnel ${ }^{12}$. SRP then can be released from its receptor such that both can now participate in further rounds of targeting ${ }^{13}$.

In eukaryotes, $\mathrm{SR}$ is composed of two subunits, the $70 \mathrm{kDa}$ peripheral membrane protein $\mathrm{SR} \alpha$ (ref. 14), which is anchored to the membrane by the $30 \mathrm{kDa} \mathrm{SR} \beta$, which has a single N-terminal transmembrane (TM) domain and short luminal domain ${ }^{15}$. Both $\mathrm{SR} \alpha$ and SR $\beta$ are GTPases; SR $\alpha$ has a bacterial homologue FtsY, and both of them share a characteristic GTPase domain (NG domain), which is also found in SRP54 and its bacterial homologue Ffh, as well as in a third member of the SRP GTPases, FlhF ${ }^{16,17}$. The NG domains of SR $\alpha$ and SRP54 interact in a GTP-dependent manner. Complex formation is kinetically accelerated by the presence of the SRP RNA and by the binding of a bona fide signal sequence ${ }^{18,19}$. Studies with the bacterial SRP-SR complex reveal that its subsequent interaction with the translocon (SecYEG) induces molecular rearrangements, which stimulate GTP hydrolysis in both GTPases leading to the release of signal sequence from SRP and its transfer to the translocon ${ }^{20}$.

$\mathrm{SR} \alpha$ is bound to $\operatorname{SR} \beta$ by its SRX domain, which possess a longin domain fold ${ }^{21,22}$. The NG and SRX domains are connected by a flexible linker, rich in charged residues, whose function is poorly characterized. FtsY lacks such linker and SRX domains, and instead possesses a natively unfolded A-domain, which is important for membrane binding via two lipid-binding motifs ${ }^{23-25}$. SR $\beta$, in contrast, is closely related to the ARF and Sar1 family of GTPases ${ }^{15,26}$. However, details of the SR $\beta$ GTPase cycle are not well understood.

Not all secretory proteins use the SRP targeting pathway; characterized first in the yeast Saccharomyces cerevisiae, an SRP-independent translocation pathway was identified that, instead of SRP, absolutely requires the ER membrane protein Sec62 (ref. 27). Sec62 associates with the same core Sec61 channel used by the SRP pathway, forming a larger Sec translocase, alongside Sec63, Sec71 and Sec72 (ref. 28). Furthermore, there is no obligate coupling of translation and targeting with this pathway, unlike with SRP-dependent targeting. Substrates can be released from the ribosome and maintained in an unfolded conformation by chaperones of the heat-shock protein 70 (Hsp70) family ${ }^{29,30}$. They can then interact with the Sec translocase, which again forms the protein-conducting channel. In this case, the action of the ER Hsp70s (Kar2 and Lhs1), which are recruited to the luminal face of the Sec translocase by Sec63, drive the energetics of the translocation reaction ${ }^{31,32}$.
The pathway a particular yeast precursor will take is largely determined by the signal sequence, with more hydrophobic signal sequences being targeted via SRP and less hydrophobic sequences translocated via the Sec62 pathway ${ }^{33}$. The Sec62 pathway is, however, not restricted to yeast; homologues of Sec62 and Sec63, but not the non-essential Sec71 or 72, are also present in higher eukaryotes and furthermore can complement deletions of their homologues in yeast; suggesting that they perform similar functions ${ }^{34-36}$.

Recently, the first substrates for the mammalian Sec62 pathway were identified and they are typically short secretory proteins including insulin, apelin and statherin ${ }^{37-39}$, as well as the insect protein preprocecropin, which was known for a long time to translocate independently of $\mathrm{SRP}^{40}$. The fact that these precursors are very short makes them poor substrates for SRP, as they are likely released from the ribosome before SRP has had a chance to bind to their signal sequence efficiently. Before binding to the translocon, some of these short secretory proteins can also interact with upstream cytosolic factors, including calmodulin and components of GET-targeting pathway, which typically delivers C-terminally anchored membrane proteins to the $\mathrm{ER}^{39,41,42}$.

Both the SRP- and Sec62-dependent pathways converge at the Sec61 translocase, but require different accessory factors to associate with Sec61. SR and ribosomes are required in the SRP pathway, and Sec62 in the SRP-independent Sec62 pathway. Evidence suggests that Sec63 is likely to be involved with both pathways ${ }^{43,44}$. At present, little is known about the dynamics and interconvertability of these complexes.

Here we have explored the interaction of both Sec62 and SR with the mammalian translocon and find that interaction of ribosomes and Sec62 with the core Sec61 translocon is mutually exclusive. Furthermore, we identify a specific role for SR, involving the $\mathrm{SR} \alpha$ linker domain, in displacement of Sec62, which provides a mechanism for the interconversion of translocons.

\section{Results}

Sec62 interacts with Sec61 in a ribosome-sensitive manner. We first compared the interaction profile of Sec61 with translocon-associated proteins in intact canine pancreatic rough microsomes (RMs), where $>80 \%$ of translocons are engaged with ribosomes ${ }^{45}$, and microsomes stripped of ribosomes by puromycin-high salt treatment (PKRMs). The compound bismaleimidohexane (BMH), a cysteine-reactive homobifunctional reagent was used to monitor crosslinking from the single endogenous cysteine in Sec61 $\beta$ (Fig. 1a). As reported previously ${ }^{46}$, the Sec61 $\beta$ cross-link profile was strikingly different for these two preparations. In particular, crosslinking between Sec61 $\beta$ and Sec62 was very weak in the RM, but dramatically increased in the PKRM.

We repeated this experiment with membranes stripped of ribosomes with EDTA and high salt (EKRM) and monitored crosslinking of Sec62 by western blot. As observed with PKRMs, there was a dramatic enhancement in Sec62-Sec61 $\beta$ crosslinking with EKRMs compared with RMs (Fig. 1b). In fact more than $50 \%$ of Sec62 could be cross-linked to Sec61 $\beta$ with EKRMs (Fig. 1b). To test whether the loss of crosslinking between these two components was specifically due to the removal of ribosomes from the membrane, purified canine salt-washed ribosomes were allowed to rebind to the EKRMs and the cross-link assay was repeated (Fig. 1b). This led to a dose-dependent reduction in crosslinking between Sec62 and Sec61 $\beta$. Hence, as reported previously ${ }^{34}$, the Sec62-Sec61 $\beta$ interaction is strongly dependent on the absence of ribosomes. 
a

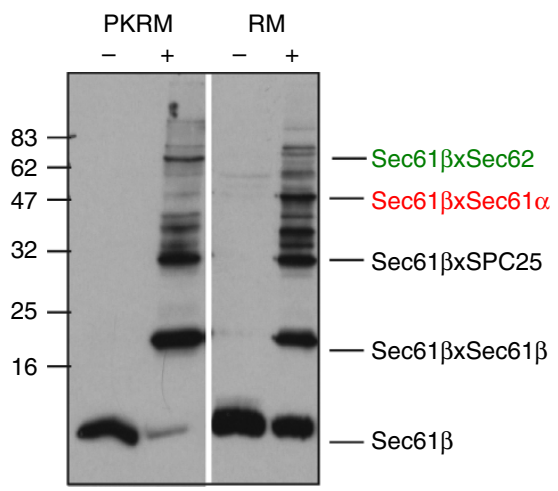

b

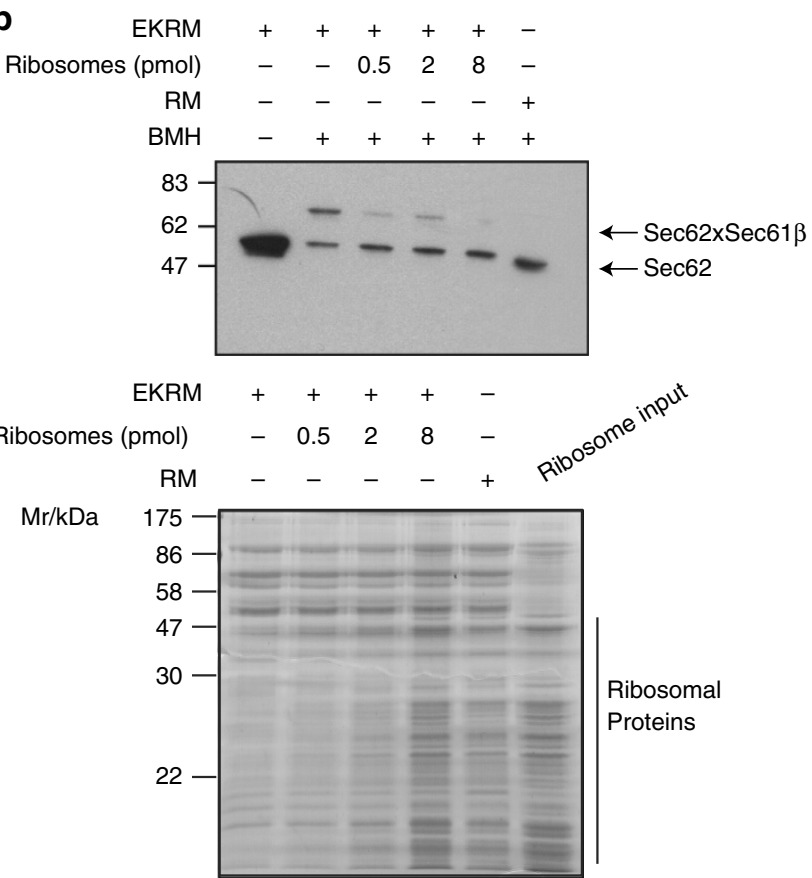

Figure 1 | Sec62 interacts with the Sec61 complex in a ribosomesensitive manner. (a) Equivalent amounts of RM and PKRM (10 eq) were treated with either DMSO or BMH $(10 \mu \mathrm{M})$ and then analysed by SDSPAGE and western blot with anst-Sec61 $\beta$ antisera. Positions of major crosslink species are indicated. (b) EKRM ( 8 eq) were incubated with increasing amounts of purified high salt-washed canine ribosomes (as indicated) before crosslinking as in a. An equivalent amount of RM were treated similarly with $\mathrm{BMH}$. Samples were then analysed by western blot with antiSec62 antibodies. The position of the major Sec62 $\times$ Sec61 $\beta$ cross-link species is indicated. Binding of ribosomes to EKRM was monitored in parallel by membrane-pelleting and analysis of the pellet fractions by SDSPAGE and staining with Coomassie Brilliant Blue.

Binding of SR to Sec61 positions SR $\alpha$ close to Sec61ß. To explore the interaction of SRP receptor with the translocon, we made use of a recombinant form of the SRP receptor, which lacks the N-terminal luminal and TM domains of SR $\beta$ (Fig. 2a), and which is functionally active in co-translational translocation ${ }^{47}$. In canine microsomes, SR is considerably sub-stoichiometric to Sec61 complex ${ }^{45}$, hence any SR-binding sites are unlikely to be saturated. Again, monitoring crosslinking from the single cysteine in Sec61 $\beta$, we assessed the effect of adding recombinant SR on the environment of the Sec61 complex (Fig. 2b, lane 7). In the case of RMs, SR $\alpha / \beta \Delta \mathrm{N}$ had little effect on the Sec61 $\beta$ crosslinking profile. In contrast, with EKRMs we observed a rather dramatic alteration in the cross-link profile on addition of SR. In particular, we observed a strong increase in a $\sim 80 \mathrm{kDa}$ cross-link species, in good agreement with the expected size of an $\operatorname{SR} \alpha \times \operatorname{Sec} 61 \beta$ adduct (Fig. 2b). This $80 \mathrm{kDa}$ band was also observed with RM but to a much lesser extent. To assess whether the $80 \mathrm{kDa}$ cross-link species observed in the presence of $S R \alpha / \beta \Delta N$ was indeed an SR $\alpha \times \operatorname{Sec} 61 \beta$ cross-link, we performed crosslinking and then reisolated recombinant $S R \alpha$ via the histidine (His)-tag using Ni-NTA resin, following membrane solubilisation (Fig. 2c). The $80 \mathrm{kDa}$ cross-link species and the weaker band above this bound to the Ni-NTA resin, unlike Sec61 $\beta$ and all the other Sec61 $\beta$ cross-link products. The presence of the double band likely arises due to crosslinking of the single cysteine of Sec61 $\beta$ to one or the other of two different cysteines within $\mathrm{SR} \alpha$ resulting in products with different electrophoretic mobility.

We also analysed cross-linked samples by western blot with Sec61 $\beta$ and SR $\alpha$ antiserum in parallel (Supplementary Fig. 1a). This clearly revealed that the strong $80 \mathrm{kDa}$ species also cross-reacts with $\mathrm{SR} \alpha$ antisera.

To test whether endogenous SR, that is, with the SR $\beta$ TM domain intact, could also be cross-linked to Sec61 $\beta$, we treated EKRM with BMH and performed denaturing immunoprecipitation with anti-SR $\alpha$ antiserum (Fig. 2d). Again, an $80 \mathrm{kDa}$ Sec61 $\beta$ cross-link species was detected and could be specifically immunoprecipitated with $\mathrm{SR} \alpha$ antibodies but not with an unrelated antiserum.

Finally, we tested whether endogenous purified SR and Sec61 complex could also interact. Sec61 complex purified from pancreatic microsomes and reconstituted alone in liposomes and treated with BMH only revealed Sec $61 \beta$ cross-links to Sec61 $\alpha$, Sec61 $\gamma$ and homodimers of Sec61 $\beta$ (Fig. 2e). In contrast, proteoliposomes that also contained purified endogenous SR and signal peptidase complex (SPC) revealed an additional albeit weak $80 \mathrm{kDa}$ Sec61 $\beta$ cross-link species in excellent agreement with the cross-link between SR $\alpha$ and Sec61 observed with recombinant SR and in EKRM.

SRP receptor blocks Sec61 $\beta \times$ Sec 62 crosslinking. In contrast to the $\operatorname{Sec} 61 \beta \times \operatorname{SR} \alpha$ cross-link species, which increased in abundance when SR was added to EKRMs, most other Sec61 $\beta$ derived cross-linked adducts were reduced, in particular, the cross-link between Sec61 $\beta$ and Sec62 (Fig. 2b). Moreover, this effect could be confirmed by pre-incubating EKRMs with increasing concentrations of SR, which revealed a dose-dependent inhibition in crosslinking between Sec62 and Sec61 $\beta$ (Fig. 3a). When this experiment was repeated using a mutant SRP receptor, which contains only the SRX domain of $\operatorname{SR} \alpha\left(\operatorname{SR} \alpha_{126} / \beta \Delta \mathrm{N}\right.$, Fig. $2 a)^{5}$, a much weaker reduction in Sec61 $\beta-S e c 62$ crosslinking was observed. This suggests that either the linker and/or NG regions are important for the inhibitory effect observed (Figs $2 \mathrm{~b}$ and $3 b$ ). Similar results were obtained using the bifunctional reagent bismaleimidoethane, which has a shorter spacer arm between the reactive maleimides than BMH (10 and $16 \AA$, respectively; Fig. 3b).

As both SR $\alpha$ and SR $\beta$ are GTPases, we also assessed whether the inhibition of the Sec61-Sec62 interaction was dependent on guanine nucleotides. However, pre-incubation of SR and EKRMs with either GDP or the non-hydrolyzable analogue GppNHp (5'-guanylyl imidodiphosphate) had no effect on the SR-induced reduction of Sec61 $\times$ Sec 62 crosslinking (Fig. 3c). Consistent with this result, crosslinking between HisSR $\alpha$ and Sec61 $\beta$ was also insensitive to guanine nucleotides (Supplementary Fig. 1b).

SR $\alpha$ linker facilitates ribosome and translocon interaction. The fact that the inhibitory effect of SR was independent of 
a

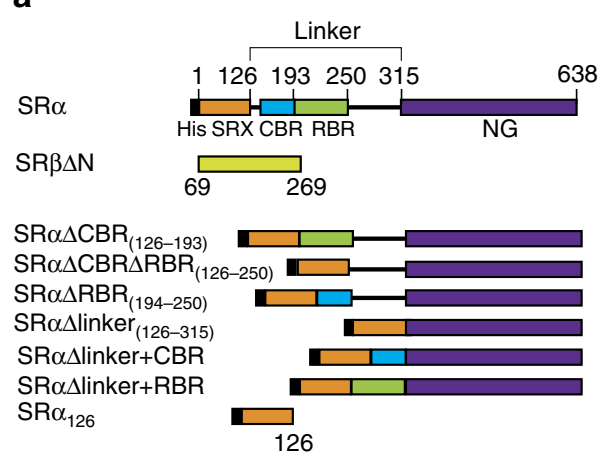

d

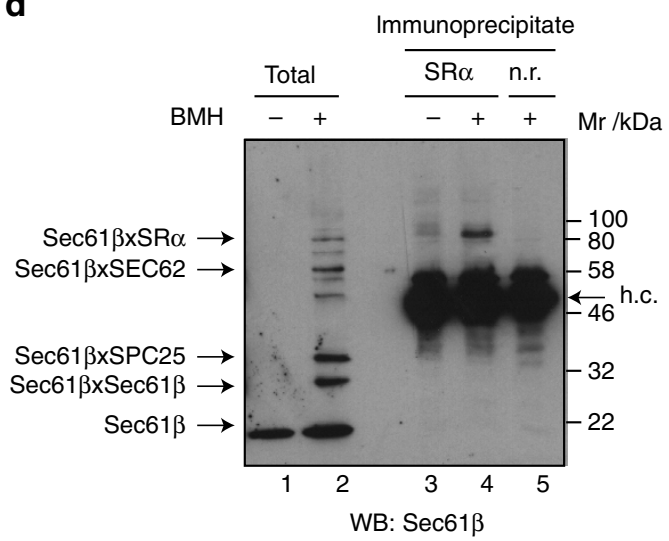

b

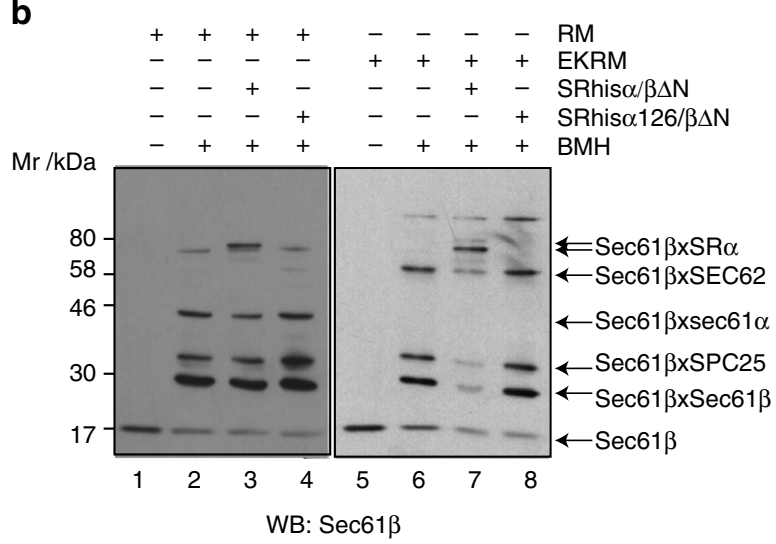

WB: Sec61ß

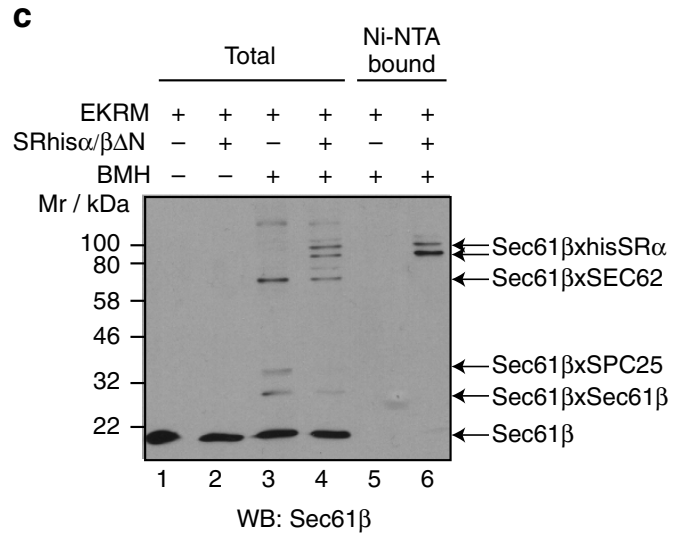

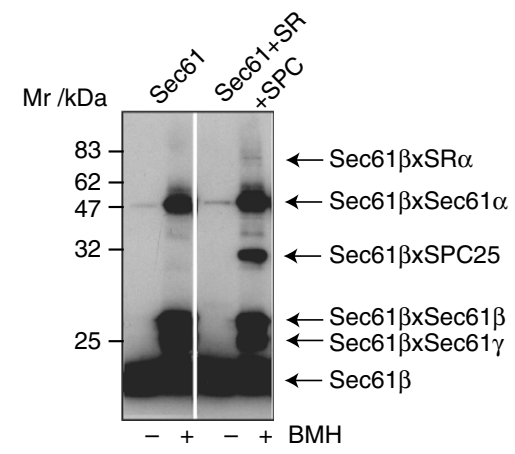

WB: Sec61ß

Figure 2 | SR binds to the translocon in close proximity to Sec61p. (a) An overview of the SR constructs used in this study. (b) RM or EKRM (32 eq) were preincubated alone or with purified recombinant SRP receptor $(7.5 \mu \mathrm{M}), \mathrm{SR} \alpha / \beta \Delta \mathrm{N}$ or a mutant $\mathrm{SR}$, lacking the entire linker and $N G$ domain of $S R \alpha\left(S R \alpha_{126} /\right.$ $\beta \Delta N$ ). Samples were then analysed by BMH crosslinking followed by SDS-PAGE and western blot with Sec61 $\beta$ antibodies. (c) EKRM preincubated with and without $\mathrm{SR} \alpha / \beta \Delta \mathrm{N}$, were treated, where indicated, with $\mathrm{BMH}(10 \mu \mathrm{M})$. Samples were then solubilized with Triton X-100 and high salt and then incubated with Ni-NTA beads to recover hisSR $\alpha$ together with any cross-link products that also contain hisSR $\alpha$. Samples were then analysed by SDS-PAGE and western blot with Sec61 $\beta$ antibodies. (d) EKRM were treated with BMH $(40 \mu \mathrm{M})$ before denaturing immuno-precipitation with either anti-SR $\alpha$ or a nonrelated antiserum (n.r.). An aliquot of the total reaction (5\%) along with the immunoprecipitated material was analysed by SDS-PAGE and western blot with Sec61ß antibodies. Positions of the cross-link products and the lgG heavy chains (h.c.) are indicated. (e) Proteoliposomes reconstituted with purified Sec61 alone or together with purified endogenous SR and signal peptidase complex (SPC) were treated with BMH (10 $\mu$ M) and analysed as in a.

nucleotides, led us to investigate whether the $\mathrm{SR} \alpha$ linker domain, rather than the GTP-binding NG domain, might be responsible. Comparison of the linker region from different eukaryotes reveals two distinct charged regions, which we term CBR and RBR (Fig. 2a, Supplementary Fig. 2). A series of mutants were generated lacking either just the first one $(\Delta \mathrm{CBR})$, both charged domains $(\triangle \mathrm{CBR} \triangle \mathrm{RBR})$ or the entire linker domain ( $\Delta$ linker), but retaining the SRX and NG domains intact (Fig. 2a).
When the cross-link assay was repeated with these mutants, in contrast to the full-length SR, none of them could reduce the Sec61 $\beta \times$ Sec62 product (Fig. 3d). These results strongly suggest that the linker domain is important for this effect. When the construct lacking the entire linker domain (residues 126-315) was used $\left(\mathrm{SR} \alpha_{\Delta \text { linker }}\right)$, an additional cross-link adduct of $92 \mathrm{kDa}$ was present corresponding precisely to the size of Sec62 plus $\mathrm{SR} \alpha_{\Delta \text { linker. }}$ 
a

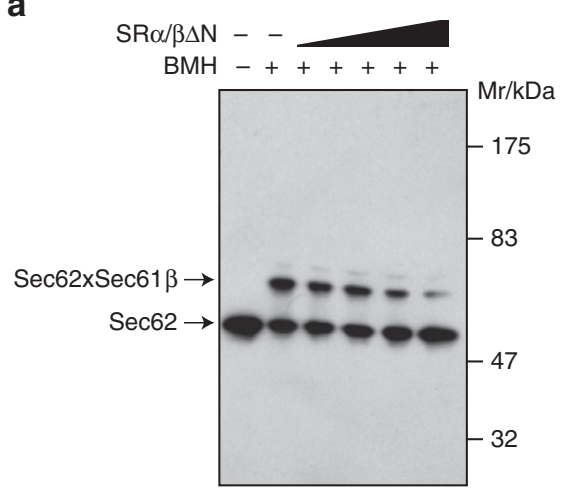

b
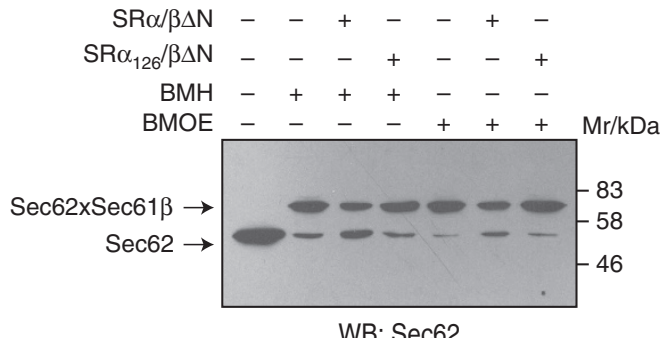

WB: Sec62

WB: Sec62

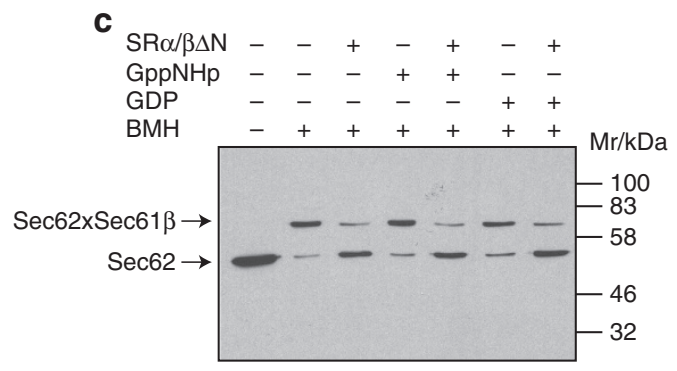

WB: Sec62

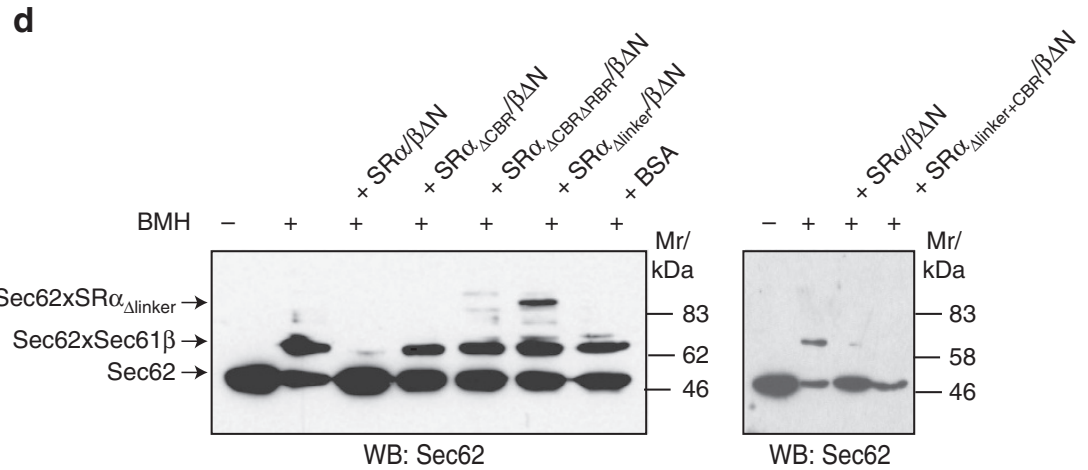

Figure 3 | SR can displace Sec62 from Sec61p. (a) EKRM (32 eq) were preincubated with increasing concentrations of $S R \alpha / \beta \Delta N(750 \mathrm{nM}, 1.5 \mu M$, $3.75 \mu \mathrm{M}$ and $7.5 \mu \mathrm{M}$ ), and then treated with BMH before analysis with SDS-PAGE and western blotting for Sec62. (b) EKRM were preincubated with either $\mathrm{SR} \alpha / \beta \Delta \mathrm{N}$ or $\mathrm{SR} \alpha_{126} / \beta \Delta \mathrm{N}$ and then treated with $\mathrm{BMH}$ or bismaleimidoethane (BMOE) as indicated and analysed as in a. (c) EKRM were preincubated either alone or with $S R \alpha / \beta \Delta N$ in the absence of nucleotide or in the presence of either $10 \mathrm{mM}$ GppNHp or $10 \mathrm{mM}$ GDP, before crosslinking with BMH and analysis as in a. (d) EKRM were incubated with $B S A, S R \alpha / \beta \Delta N$ or $S R \alpha / \beta \Delta N$ harbouring the indicated linker mutations and then crosslinking was induced with BMH before analysis with SDS-PAGE and western blotting with anti-Sec62 antisera. The position of a novel cross-link observed between Sec62 and $\mathrm{SR} \alpha_{\Delta \text { linker }}$ is indicated.

To test whether the CBR region is sufficient for this effect, we performed the same experiment with a construct where the entire linker is replaced with just the CBR ( $\Delta$ linker + CBR) (Fig. $3 \mathrm{~d})$. Indeed this construct was also able to reduce crosslinking between Sec61 $\beta$ and Sec62. Our data show that the first charged region is required for Sec62 displacement.

We also monitored membrane binding of the different SR linker mutants using EKRM in a floatation assay (Supplementary Fig. 3). Constructs with CBR (that is, full-length SR and $\Delta$ linker $+\mathrm{CBR}$ ) showed the strongest membrane binding, consistent with the role of this domain in interacting with the translocon. Although the other mutants showed lower membrane binding, all of them could still interact with the membrane. In the case of the $\Delta$ linker construct, this is in good agreement with the observed cross-link between $\operatorname{SR} \alpha_{\Delta \text { linker }}$ and Sec62. This indicates that the inability of mutants lacking the CBR domain to perturb the Sec62-Sec61 interaction is not simply due to a loss of membrane or translocon interaction.

SR $\alpha$ linker domain is important for ribosome binding of SR. The linker domain is also known to be required for ribosome binding of mammalian $\mathrm{SR}^{47,48}$. We made use of the linker mutants to assess whether this property of SR might also depend on a specific region of the linker domain. Using a sedimentation assay with salt-washed canine pancreas ribosomes, binding of full-length SR to ribosomes could be observed as reported previously (Fig. 4a) ${ }^{47,48}$. Removal of both charged regions $(\triangle \mathrm{CBR} \triangle \mathrm{RBR})$ or the entire linker ( $\Delta$ linker) abolished ribosome binding; however, deletion of only the $\mathrm{N}$-terminal region $(\triangle \mathrm{CBR})$ led to increased ribosome association. Deletion of just the RBR domain strongly reduced ribosome binding. In contrast, a 
a

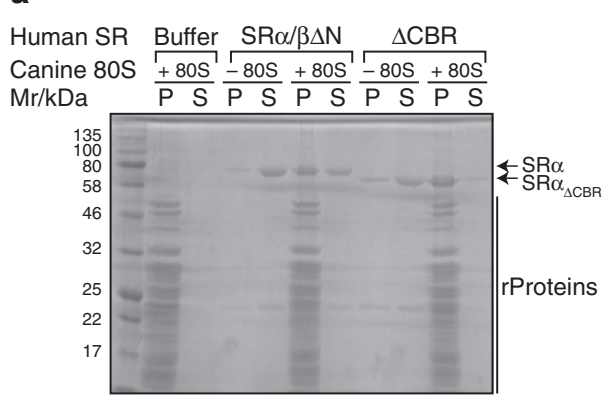

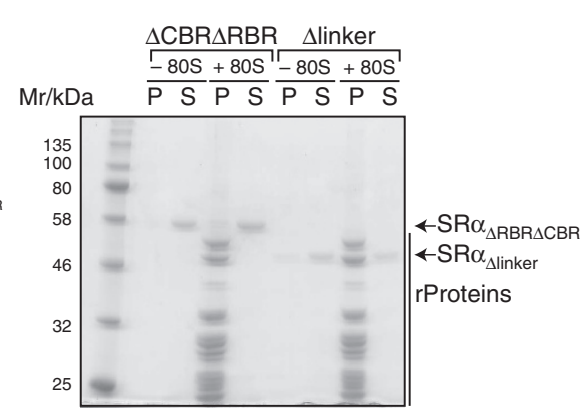

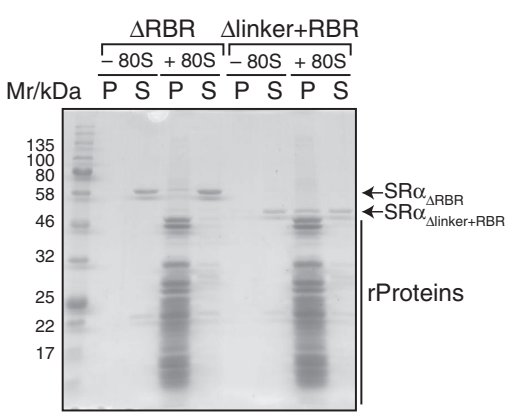

b
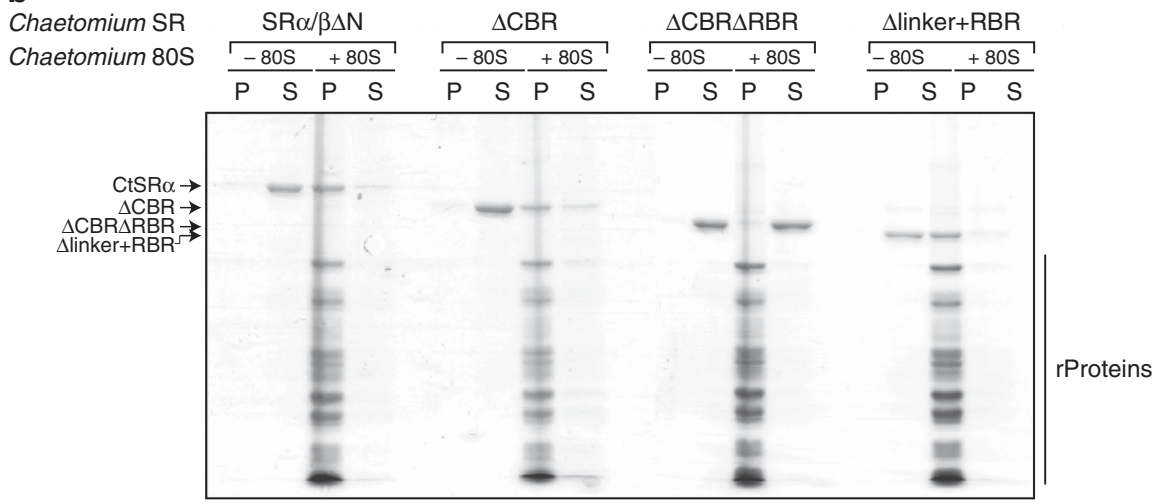

Figure 4 | The conserved charged region of the SR $\alpha$ linker domain is involved in ribosome binding. (a) Indicated constructs of human $S R \alpha / \beta \Delta N$ were incubated in the presence and absence of salt-washed canine pancreatic ribosomes. Bound material was then recovered by sedimentation through a sucrose cushion and the supernatant $(S)$ and pellet $(P)$ fractions analysed by SDS-PAGE and staining with Coomassie Brilliant Blue. (b) Analysis as in a but with salt-washed ribosomes and SR $\alpha$ constructs from Chaetomium thermophilum (Ct).

construct where the entire linker is replaced with the RBR domain alone $(\Delta$ linker $+\mathrm{RBR})$ still showed ribosome association, albeit not as efficient as full-length SR.

While the ability of SR to bind ribosomes appears linked to the RBR region of the linker domain, this contrasts to the effect of SR on Sec61 $\beta \times$ Sec62 crosslinking, which is closely linked to the CBR region (Fig. 3d). Hence the linker domain appears to be involved in both binding the ribosome and destabilizing the Sec62-Sec61 interaction. However, these two functions require distinct regions of the linker domain.

SR ribosome binding is evolutionarily conserved. Having observed that the linker domain harbours two distinct charged regions, we used multiple sequence alignments to further dissect these elements (Supplementary Fig. 2a). The CBR, which is involved in Sec62 displacement, is more specific to animals, while the RBR is evolutionarily well-conserved between fungi, plants and animals. As the latter was implicated in ribosome binding, the role of this region was further assessed using a fungal SR. Analogous linker domain mutations were generated in SR $\alpha$ from the thermophilic fungus Chaetomium thermophilum (Supplementary Fig. 2b) and their ability to bind to Chaetomium $80 \mathrm{~S}$ ribosomes was tested (Fig. $4 \mathrm{~b}$ ). Compared with human $\mathrm{SR} \alpha$, full-length $\operatorname{CtSR} \alpha$ is more stable in the absence of $\operatorname{SR} \beta$ and allowed us to monitor ribosome binding independent of SR $\beta$. Moreover, we recently showed that while $\operatorname{CtSR} \alpha / \operatorname{SR} \beta \Delta \mathrm{N}$ can bind ribosomes, both $\operatorname{CtSR} \beta \Delta \mathrm{N}$ alone and the minimal $\operatorname{CtSR} \alpha_{138} /$ $\mathrm{SR} \beta \Delta \mathrm{N}$ complex are unable to bind ribosomes ${ }^{26}$. Full-length $\mathrm{CtSR} \alpha$ alone readily bound to the ribosomes in a sedimentation assay (Fig. 4b), as well as to canine ribosomes (Supplementary
Fig. 4b). Deletion of the non-conserved linker region ( $\triangle \mathrm{CBR})$ had very little effect on ribosome binding. In contrast, deletion of the conserved RBR region as well almost completely abolished ribosome binding $(\triangle \mathrm{CBR} \triangle \mathrm{RBR})$. Replacing the entire linker with just the RBR domain completely restored ribosome binding $(\Delta$ linker $+\mathrm{RBR})$. Hence the role of the RBR is conserved between yeast and mammals.

Unlike the ribosome-binding activity of $\mathrm{SR} \alpha, \mathrm{CtSR} \alpha$ was unable to destabilize the interaction between Sec61 $\beta$ and Sec62 (Supplementary Fig. 4c), suggesting that this interaction is specific to higher eukaryotes. Interestingly, the $\mathrm{CtSR} \alpha \mathrm{NG}$ domain alone could also be cross-linked to Sec62, as was observed with the human $\mathrm{SR} \alpha_{\Delta \text { linker }} / \beta \Delta \mathrm{N}$ construct (Supplementary Fig. 4c), indicating that the NG-translocon interaction is conserved.

SR inhibits translocation of Sec62-dependent substrates. Having observed that human SR can perturb the association between mammalian Sec62 and Sec61, the functional effect of SR on the translocation of Sec62-dependent substrates was tested. Insect preprocecropin A has long been known to translocate in an SRP-independent manner and was recently shown to instead require Sec62 (refs 37,49). Similarly, the small mammalian secretory proteins apelin and statherin also translocate in a Sec62-dependent manner ${ }^{49}$. Apelin, statherin and preprocecropin A derivatives, possessing a $\mathrm{N}$-glycosylation opsin tag to monitor ER translocation, were synthesised in vitro in rabbit reticulocyte lysate in the presence of labelled methionine. To exclude the possibility of any co-translational translocation, the translation reaction was treated with puromycin before the addition of RMs in 
the presence or absence of $S R \alpha / \beta \Delta N$. Precursor translocation was then monitored via $\mathrm{N}$-linked glycosylation. All three substrates showed a marked decrease in translocation in the presence of SR $\alpha /$ $\beta \Delta \mathrm{N}$ (Fig. 5a). In contrast, the $\mathrm{C}$ terminus of the tail-anchored membrane protein cytochrome B5-opsin construct, which can insert into the ER membrane spontaneously ${ }^{50}$, was N-glycosylated equally efficiently in the presence or the absence of SR, indicating that the loss of N-linked glycosylation of the short secretory proteins is not due to an indirect effect of SR on oligosaccharyltransferase activity.

To further investigate the specificity of this perturbation, we compared the inhibitory effect of the different SR linker deletion constructs on preprocecropin translocation. We performed the analysis in a co-translational reaction so that we could monitor effects on the SRP-dependent translocation of preprolactin in parallel (Fig. 5b,c). Control reactions performed in the absence of membranes or in the presence of membranes followed by treatment with Endo $\mathrm{H}$ confirmed the identity of the translocated species (Supplementary Fig. 5).

As with the post-translational assay, full-length SR led to a significant inhibition of preprocecropin translocation. In contrast, the $\Delta \mathrm{CBR}, \Delta \mathrm{CBR} \Delta \mathrm{RBR}$ and $\Delta$ linker constructs all failed to block translocation. The construct where the linker is replaced by the CBR domain alone $(\Delta$ linker + CBR) also inhibited translocation, although not quite as strongly as full-length SR. These results are, therefore, in close agreement with the ability of the different linker mutants to effect the Sec61-Sec62 interaction.

Full-length SR was also able to reduce translocation of preprolactin, although the effect was much weaker than observed for preprocecropin. This is consistent with previous studies using endogenous SR and Sec61 complex reconstituted into proteoliposomes, which also showed that increasing levels of SR relative to Sec61 reduced translocation of the efficiency of preprolacti ${ }^{45}$.

Importantly, none of the linker mutants were able to inhibit preprolactin translocation. The lack of effect of the $\Delta$ linker + CBR construct, which did block preprocecropin translocation, therefore indicates distinct underlying mechanisms of inhibition for the two pathways. Neither the full-length SR nor any of the linker mutants had any effect on cytochrome B5 integration.

\section{Discussion}

Here we have shown that the interaction of Sec62 and Sec61 as revealed by crosslinking is highly sensitive to the presence and the absence of ribosomes. In contrast to the core Sec61 heterotrimer, the heptameric yeast Sec complex has been shown to be unable to bind to ribosomes ${ }^{51}$. Consistent with this, low resolution cryoelectron microscopy structures of the Sec complex reveal additional density above the cytoplasmic face of the core Sec61 heterotrimer ${ }^{52}$, which would likely occupy a similar position as the ribosome $e^{53}$ and hence occlude binding. This is in good agreement with the observation that crosslinking between mammalian Sec62 and Sec61 $\beta$ is strongly induced by the removal of ribosomes from RMs and is inhibited by their readdition. Furthermore, cross-links between Sec62 and Sec61 $\beta$ were shown previously to be exclusively absent from the ribosome-associated membrane protein fraction following detergent solubilisation ${ }^{34,35}$.

a
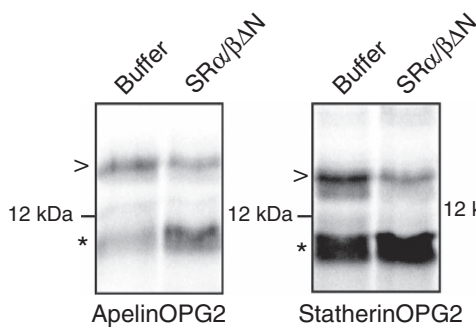

b

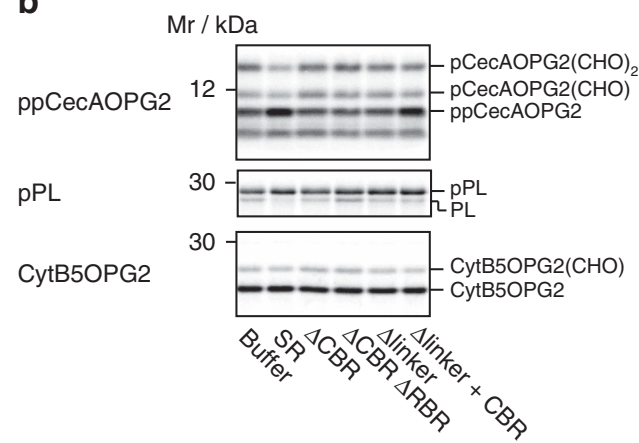

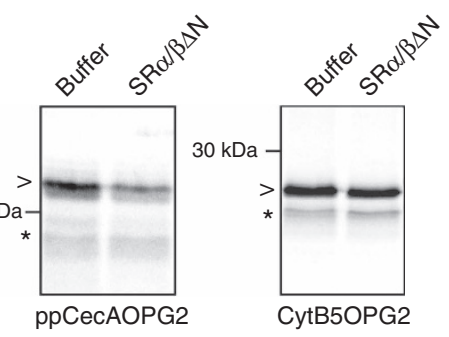

C

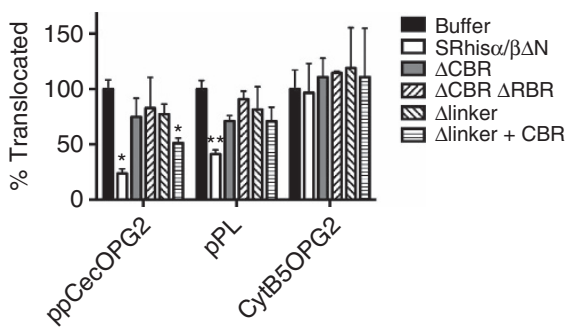

Figure 5 | SR can inhibit translocation of Sec62-dependent precursors. (a) Constructs of apelin, statherin, preprocecropin A (ppcec A) and cytochrome B5 (cyt B5) each with a C-terminal opsin tag containing two N-linked glycosylation sites (OPG2) were translated in vitro in rabbit reticulocyte lysate in the presence of $\left[{ }^{35} \mathrm{~S}\right]$ methionine. Synthesis was terminated with puromycin to ensure release of all nascent chains from the ribosome. PKRM were then added in the presence of absence of $\mathrm{SR} \alpha / \beta \Delta \mathrm{N}(10 \mu \mathrm{M})$ and then incubated at $30^{\circ} \mathrm{C}$ to permit targeting and translocation. Membranes were then reisolated through a sucrose cushion and analysed by SDS-PAGE and phosphorimaging. The position of unglycosylated non signal-sequence cleaved $\left({ }^{\star}\right)$ and signalsequence cleaved, twice glycosylated species $(>$ ) is indicated. (b) Preprocecropin A (ppCec A) and cytochrome B5 both with a C-terminal opsin tag (OPG2) as well as preprolactin ( $\mathrm{pPL}$ ) were translated in reticulocyte lysate in the presense $\left[{ }^{35} \mathrm{~S}\right.$ ] methionine and microsomes that had been preincubated with either buffer or the different SR constructs. Processed and non-processed forms of each precursor were recovered by denaturing immuno-precipitation and analysed by SDS-PAGE and phosphorimaging. (c) Relative translocation efficiency was determined from the ratio of processed to non-processed form for each precursor (as in b). Translocation in the absence of recombinant SR was set to 100\%. Data are the means of three independent experiments. Error bars represent s.e.m. Differences significant from the buffer control are indicated (one-way analysis of variance, ${ }^{\star} P<0.05$, ${ }^{\star \star} P<0.01$ ). 
The fact that more than $50 \%$ of Sec62 can be cross-linked to Sec61, following removal of ribosomes, suggests that the interaction of these components is strongly favoured if ribosomes are absent. Furthermore, if the free Sec complex is unable to interact with ribosomes ${ }^{51}$, this raises the question as to how ribosomes can subsequently rebind to such complexes. Here we find that SR is able to destabilize the interaction between Sec62 and Sec61, which we detect as a loss of crosslinking between Sec62 and Sec61 $\beta$ and a block in the translocation of Sec62dependent substrates.

It is well established that SR catalyses the transfer of incoming ribosome-nascent chain complexes from SRP to the Sec61 translocon ${ }^{11}$. Hence, the ability of SR to interact with Sec61 and displace or reposition Sec62, immediately suggests a mechanism by which the Sec complex can be reorganized to permit the subsequent docking of the ribosome-nascent chain complex (Fig. 6). Furthermore, the SR-Sec61 intermediate would preferentially recruit SRP-bound ribosome-nascent chain complexes, potentially providing an additional step where targeting fidelity can be enhanced. Moreover, this would also rationalize the previous observation that SRP-bound ribosomenascent chains can access a pool of Sec61 to which free ribosomes are unable to bind ${ }^{54}$.

A recent study showed that arresting translocation of a cotranslational substrate using a small, tightly folded domain at the luminal side of the translocon, led to the stable recruitment of Sec62 (ref. 55). Hence, although dispensable for co-translational translocation of most substrates ${ }^{37-39}$, a subset of nascent chains may require Sec62 to facilitate later steps of their the translocation. Such nascent chains may possess particular features, which initiate reorganization of the ribosometranslocon complex and thereby expose a stable binding site for Sec62.

Our experiments revealed that crosslinking of $\operatorname{SR} \alpha$ to Sec61 $\beta$ was much stronger with PKRMs as compared with RMs. There is still a low amount of $\operatorname{SR} \alpha$ to Sec61 $\beta$ crosslinking in $\mathrm{RM}$, rationalised by the fact that $\sim 80-90 \%$ of translocons are engaged with ribosomes ${ }^{45}$, hence a small population of translocons are still available to bind SR.

SR also had little effect on the overall cross-link profile of Sec61 $\beta$ with RMs as compared with PKRMs. This suggests that once the ribosome is fully engaged with the translocon, access of SR is likely to be strongly hindered. This is rationalized by cryoelectron microscopy structures of the ribosome-Sec61 (ref. 53) and ribosome-SRP-SR ${ }^{6}$ complexes, which predict a strong potential overlap of density at the ribosomal exit tunnel.
Complete deletion of the linker domain led to the formation of a novel cross-link between $\operatorname{SR} \alpha$ and Sec62. This is also observed with the CtSR $\alpha$ NG domain, indicating that this interaction from the NG domain appears conserved. Human and Chaetomium NG domains both contain multiple cysteines but only one of these is conserved and is located in helix 3 of the $\mathrm{N}$-domain, hence this is a strong candidate to be involved in crosslinking to Sec62. Escherichia coli FtsY, which lacks the linker domain, is known to interact with cytoplasmic loop 5 of SecY via its NG domain. Therefore, in the absence of the linker, $\operatorname{SR} \alpha$ may also bind to the Sec61 complex in a similar manner, positioning the NG domain close to Sec62. If the linker is still present, but unable to displace Sec62, as with full-length $\mathrm{CtSR} \alpha$, this may interfere with the binding of NG domain.

As well as interacting with the translocon, $\mathrm{SR} \alpha$ also binds to the ribosome $e^{47,48}$. Our study characterizes a long stretch of positively charged residues in the linker region of eukaryotic $\mathrm{SR} \alpha$ of previously unknown function. We identify two distinct elements within the linker and show that ribosome binding requires the second element (RBR) that is conserved between fungi and mammals. Notably, a large number of factors that act on the nascent polypeptide use the tunnel exit as a hub for interaction. Although these factors are unrelated in structural and functional terms, they seem to use a common mechanism for ribosome interaction involving a stretch of positively charged residues ${ }^{56,57}$. The RBR identified in this study within the linker region of $\mathrm{SR} \alpha$ probably acts by the same mechanism.

The first element (CBR) being unique to higher eukaryotes and rich in lysine residues is necessary and sufficient for the displacement of Sec62 by SR $\alpha$. Sec62 contains several positively charged regions also rich in lysine residues. Hence the linker of $\mathrm{SR} \alpha$ and Sec62 are likely to compete for Sec translocon binding via electrostatic interactions. Indeed, Sec63 has a very negatively charged $C$ terminus ${ }^{34,35}$ and so might well contribute to the mutually exclusive binding site. In this respect, CBR and Sec62 might also compete for binding to Sec63.

Interestingly, mammalian Sec62 differs from its yeast homologues by the presence of an additional positively charged region in the N-terminal region ${ }^{58}$ (Supplementary Fig. 6). This might represent an extra level of regulation in higher eukaryotes, and explain why the additional positive-charged (CBR) region within the linker domain of human SR is required to displace Sec62 from Sec61.

Taken together, we have identified two distinct interaction motifs in SR and show that one of them serves a function that is distinct from the 'canonical' eukaryotic RBR in regulating association of Sec62 with Sec61 in the ER membrane. Cells

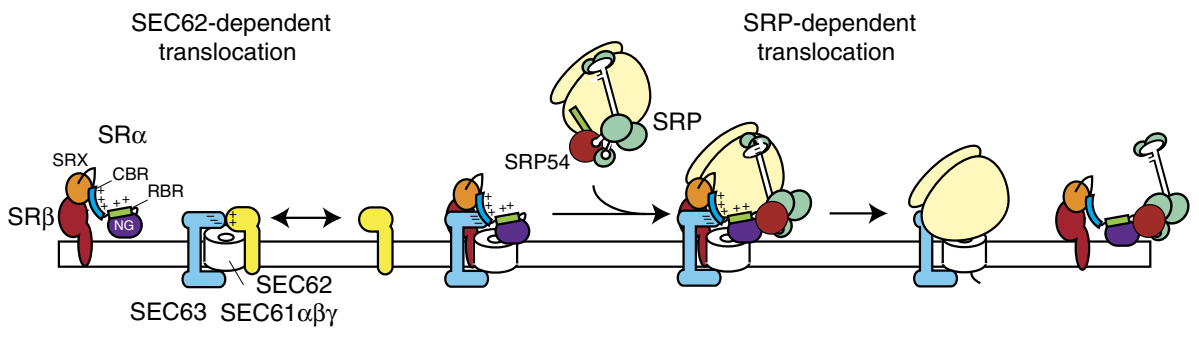

Figure 6 | Model depicting distinct roles of SR $\boldsymbol{\alpha}$ linker domain in Sec62 displacement from Sec61 and ribosome recruitment. During Sec62-dependent translocation cytosolic chaperones maintain the precursor in an unfolded translocation-competent state (upper panel). Precursors are delivered to the Sec translocon comprising Sec61 complex associated with Sec62 and Sec63. The latter are thought to interact with one another electrostatically via charged domain within the two proteins. Translocation is driven by ATP hydrolysis of the luminal Hsp70 chaperones. SRP-RNC complexes are unable to interact with the larger Sec translocon. During SRP-dependent translocation, SR can interact with the Sec translocase displacing Sec62 (lower panel). This requires the CBR of the SR $\alpha$ linker domain, which may compete with Sec62 for binding to the charged regions of Sec63 and/or Sec61. This displacement of Sec62 allows approach by RNC-SRP complexes, which can bind the SRP receptor via interactions of the NG domains of SRP54 and SR $\alpha$ and components of the ribosome with the charged RBR domain of the SR $\alpha$ linker region. Hence SR can preferentially recruit SRP-RNC complexes and then transfer them to the Sec61 translocon. 
under different physiological conditions can express different levels of $\mathrm{SR}^{59}$. In combination with our findings, this may provide a mechanism to differentially modulate the flux of substrates through two distinct ER translocation pathways, allowing cells to efficiently respond to changing profiles of secretory substrates with distinct properties under different physiological conditions.

\section{Methods}

Purification of mammalian SR and deletion variants. For purification of mammalian SR, a bi-cistronic construct containing human $\mathrm{SR} \alpha$ and murine $\mathrm{SR} \beta \Delta \mathrm{TM}$ was cloned in the pET16b vector (Novagen). Linker deletion variants of SR were created using the same construct. Expression was performed in E. coli BL21 (DE3) cells with overnight induction with $1 \mathrm{mM}$ isopropyl- $\beta$-D-thiogalactoside at $16^{\circ} \mathrm{C}$. The cells were lysed using a sonicator followed by micro fluidizer in SR buffer (25 mM HEPES pH (7.5), $300 \mathrm{mM} \mathrm{KOAc}$, and $\left.5 \mathrm{mM} \mathrm{Mg}(\mathrm{OAc})_{2}\right)$ and cleared by centrifugation at $63,000 \mathrm{~g}$ for $30 \mathrm{~min}$ using a JA25.50 (Beckman) rotor. The resulting supernatant was purified first by Ni-NTA affinity chromatography. The eluate was applied to a Q-sepharose column and the flow-through, then bound to SP-sepharose resin and eluted with a $0.2-1 \mathrm{M}$ KOAc salt gradient in SR buffer. Finally, the SR was purified by size exclusion chromatography on a Superdex 200 column ${ }^{47}$. The final concentration of salt was reduced to $150 \mathrm{mM}$ before snap freezing in liquid nitrogen.

Purification of CtSR and linker deletion variants. CtSR $\alpha$ and the deletion variants and $\operatorname{CtSR} \beta$ were cloned as hexa-histidine-tagged proteins via the $\mathrm{pETHis}$ vector $^{26,60}$. Untagged CtSR $\beta \Delta \mathrm{TM}$ was cloned into the pET24a vector (Novagen). CtSR His-tagged CtSR $\alpha$ and untagged $\mathrm{CtSR} \beta \Delta \mathrm{TM}$ were expressed individually using the auto-induction method in E. coli BL21 (DE3) cells at $24^{\circ} \mathrm{C}$ overnight ${ }^{61}$. Pellets of cells expressing $\operatorname{CtSR} \alpha$ and $\mathrm{CtSR} \beta \Delta \mathrm{TM}$ were lysed together in the lysis buffer (20 mM Tris pH 8.0, $150 \mathrm{mM} \mathrm{NaCl}, 5 \mathrm{mM} \mathrm{MgCl}_{2}, 20 \mathrm{mM}$ imidazole and $0.02 \%(\mathrm{v} / \mathrm{v})$ Nonidet-P40 ) using a microfluidizer. After the lysis, the cell debris was removed by centrifugation at $67,000 \mathrm{~g}$ using JA25.50 (Beckman) rotor. The supernatant was collected and incubated at $4{ }^{\circ} \mathrm{C}$ for $1 \mathrm{~h}$ on a rotating wheel. The CtSR complex was then purified as described for mammalian $\mathrm{SR}^{47}$. His-tagged $\operatorname{CtSR} \alpha, \operatorname{CtSR} \alpha$ deletion variants and CtSR $\beta \Delta \mathrm{TM}$ were expressed and purified similarly.

Microsome crosslinking in the presence of SR. RMs, EKRMs and PKRMs were prepared from canine pancreas ${ }^{62,63}$. Pancreatic tissue was cut into small pieces, passed through a tissue press and then homogenized in RM buffer $(20 \mathrm{mM}$ HEPES-KOH pH 7.6, $50 \mathrm{mM}$ KOAc, $2 \mathrm{mM} \mathrm{Mg}(\mathrm{OAc})_{2}, 250 \mathrm{mM}$ sucrose and $2 \mathrm{mM}$ DTT). The resulting extract was then centrifuged sequentially at $1,000 \mathrm{~g}$ and $10,000 \mathrm{~g}$ to yield a post-mitochondrial supernatant, which was overlayed onto step gradients of 1.5, 1.75 and $2 \mathrm{M}$ sucrose (in RM buffer) and centrifuged overnight at $235,000 \mathrm{~g}$ at $4{ }^{\circ} \mathrm{C}$ using a Ti45 rotor (Beckman). Membranes banding at the 1.75-2 M cushion interface were collected, diluted with RM buffer without sucrose $(\mathrm{RM}-)$ and pelleted before resuspension in RM buffer at a concentration of 4 eq $\mu l^{-1}$ to yield RMs.

EKRMs were prepared by washing RMs with high salt (650 mM KOAc) before resuspension in $20 \mathrm{mM}$ HEPES-KOH pH 7.6, $20 \mathrm{mM}$ EDTA, $650 \mathrm{mM} \mathrm{KOAc}$, $2 \mathrm{mM}$ DTT and $2 \mathrm{M}$ sucrose. Membranes were held on ice for $30 \mathrm{~min}$ and then overlayed with sucrose cushions of $1.5,1$ and $0.25 \mathrm{M}$ in the same buffer and centrifuged overnight in the SW40 rotor (Beckman) at 38,000 r.p.m. Membranes floating at the $0.25-1 \mathrm{M}$ interface were collected, diluted with $\mathrm{RM}(-)$ buffer and collected by centrifugation before resuspending in RM buffer to give EKRM. PKRM were prepared in an identical manner, except salt-washed membranes were resuspended in $20 \mathrm{mM}$ HEPES- $\mathrm{KOH}$ pH 7.6, $500 \mathrm{mM} \mathrm{KOAc}, 5 \mathrm{mM} \mathrm{Mg}(\mathrm{OAc})_{2}$, $2 \mathrm{mM}$ puromycin, $2 \mathrm{mM}$ GTP and $2 \mathrm{M}$ sucrose (prewarmed to $25^{\circ} \mathrm{C}$ ), and incubated at $25^{\circ} \mathrm{C}$ for $20 \mathrm{~min}$ before floatation.

To purify translocon components, RMs were pre-extracted with $0.1 \%(\mathrm{w} / \mathrm{v})$ digitonin and then solubilised with $3 \%(\mathrm{w} / \mathrm{v})$ digitonin in the presence of $500 \mathrm{mM}$ KOAc. The resulting extract was then centrifuged to yield a ribosome pellet and supernatant. To purify SPC, the supernatant was applied to a concanavilin A column. Bound proteins were eluted with $\alpha$-methylmannoside and then SPC purified on an SP-sepharose ion exchange column ${ }^{45}$. SR was purified from the flow-through of the concanavalin A column using an anti-SR $\alpha$ immunoaffinity column ${ }^{45,64}$. Sec61 was purified from the ribosome pellet fraction by ion-exchange chromatography with Q and SP-sepharose, following release from the ribosome by puromycin treatment ${ }^{45,64}$.

Purified components were detergent exchanged from digitonin into $0.2 \%(\mathrm{w} / \mathrm{v})$ deoxyBigCHAP on a SP-sepharose column and reconstituted into liposomes by combining with $10 \mathrm{mg} \mathrm{ml}^{-1}$ phosphatidlycholine:phosphatidlyethanolamine (4:1) and incubated overnight with SM2 Biobeads (Bio-Rad) ${ }^{45,64}$.

Ten equivalents of EKRMs or PKRMs were incubated with $0.7-10 \mu \mathrm{M}$ of mammalian SR and linker deletion variants in a $20 \mu \mathrm{l}$ reaction in the assay buffer (25 mM HEPES-KOH pH 7.5, $120 \mathrm{mM} \mathrm{KOAc,} 2 \mathrm{mM} \mathrm{Mg}(\mathrm{OAc})_{2}, 250 \mathrm{mM}$ sucrose) at $25^{\circ} \mathrm{C}$ for $10-15 \mathrm{~min}$. $\mathrm{BMH}$ was added to the final concentration of $10 \mu \mathrm{M}$ and the reaction mixture was further incubated at $25^{\circ} \mathrm{C}$ for $10 \mathrm{~min}$. The crosslinking reaction was stopped by the addition of $1 \mathrm{mM}$ DTT and incubating the sample on ice for $15 \mathrm{~min}$. The whole sample was then mixed with SDS sample buffer and proteins and crosslinking adducts were analysed by SDS-PAGE and western blotting with either Sec61 $\beta(1: 3,000), \operatorname{SR} \alpha(1: 1,000)$ or Sec62 $(1: 1,000)$ antibodies. Complete blot images for cropped panels are shown in Supplementary Fig. 7.

For immunoprecipitation of endogenous cross-linked SR $\alpha$, EKRM (100 eq) were treated with $40 \mu \mathrm{M} \mathrm{BMH}$ for $10 \mathrm{~min}$ at $30^{\circ} \mathrm{C}$. After quenching with $10 \mathrm{mM}$ DTT, membranes were reisolated by centrifugation and then resuspended in $70 \mu \mathrm{l}$ of $20 \mathrm{mM}$ Tris- $\mathrm{HCl} \mathrm{pH} \mathrm{8.0.} \mathrm{2 \%} \mathrm{(w/v)} \mathrm{SDS} \mathrm{and} 2 \mathrm{mM}$ EDTA and heated for $10 \mathrm{~min}$ at $70{ }^{\circ} \mathrm{C}$. The denatured membranes were diluted with $700 \mu \mathrm{l}$ of ice-cold immunoprecipitation (IP) buffer A (20 mM Tris- $\mathrm{HCl}$ pH 8.0, 0.4\% (v/v) NonidetP40, $150 \mathrm{mM} \mathrm{NaCl}, 1 \mathrm{mM}$ EDTA, $1 \mathrm{mM}$ PMSF (phenylmethylsulfonyl fluoride)) and then $2 \mu \mathrm{l}$ of anti-SR $\alpha$ or a non-related rabbit antiserum were added. After rolling for $1 \mathrm{~h}$ at $4{ }^{\circ} \mathrm{C}$, immune complexes were recovered with protein A-sepharose (Genscript) and then washed two times with IP buffer A, two times with $20 \mathrm{mM}$ Tris- $\mathrm{HCl} \mathrm{pH} 8.0,0.2 \%(\mathrm{v} / \mathrm{v})$ Triton X-100, $500 \mathrm{mM} \mathrm{NaCl}, 1 \mathrm{mM}$ EDTA and once with $10 \mathrm{mM}$ Tris- $\mathrm{HCl} \mathrm{pH} \mathrm{8.0,} \mathrm{before} \mathrm{elution} \mathrm{with} \mathrm{SDS-PAGE} \mathrm{sample} \mathrm{buffer} \mathrm{at}$ $70^{\circ} \mathrm{C}$ for $10 \mathrm{~min}$. Samples were then analysed by SDS-PAGE and western blot with Sec61 $\beta$ antibodies $(1: 1,000)$ and detection with protein A-horseradish peroxidase (Sigma) to reduce cross-reaction with IgG heavy chains.

Preparation of Canine and Chaetomium 80 S ribosomes. To prepare canine ribosomes ${ }^{47}$, dog RM were resuspended in 50 mM HEPES-KOH pH 7.6, $600 \mathrm{mM}$ KOAc, $12 \mathrm{mM} \mathrm{Mg}(\mathrm{OAc})_{2}, 2 \mathrm{mM} \mathrm{DTT}$, with protease inhibitor cocktail and treated with $0.5 \mathrm{mM}$ puromycin for $15 \mathrm{~min}$ at $25^{\circ} \mathrm{C}$. The reaction was then overlayed onto a $1.5 \mathrm{M}$ sucrose cushion made up in the same buffer and centrifuged at $444,000 \mathrm{~g}$ for $1 \mathrm{~h} 20 \mathrm{~min}$ at $15^{\circ} \mathrm{C}$ in the MLA-80 rotor (Beckmann). The resulting ribosomal pellet was then rinsed and resuspended in $25 \mathrm{mM}$ HEPES-KOH pH 7.6, $120 \mathrm{mM}$ KOAc, $2 \mathrm{mM} \mathrm{Mg(OAc})_{2}, 1 \mathrm{mM}$ DTT.

For purification of Chaetomium ribosomes ${ }^{56}$, C. thermophilum cells were grown in a rotary shaker at 90 r.p.m. at $55^{\circ} \mathrm{C}$ for 3 days. Cells were then harvested with a vacuum filter and ground to a fine powder in a mortar in the presence of liquid nitrogen. The powdered mycelium was then resuspended in $20 \mathrm{mM}$ HEPES-KOH ( $\mathrm{pH}$ 7.5), $100 \mathrm{mM}$ potassium acetate, $125 \mathrm{mM}$ sucrose, $7.5 \mathrm{mM} \mathrm{Mg}(\mathrm{OAc})_{2}, 1 \mathrm{mM}$ DTT and $0.5 \mathrm{mM}$ PMSF and centrifuged at $28,000 \mathrm{~g}$ in SS-34 rotor (Sorvall), for $15 \mathrm{~min}$ to remove the insoluble material. To pellet ribosomes, the supernatant was overlaid on a high-salt sucrose cushion ( $500 \mathrm{mM}$ potassium acetate, $1.5 \mathrm{M}$ sucrose) prepared in the same lysis buffer and centrifuged at $300,000 \mathrm{~g}$ in a Ti70 rotor (Beckman) for $18 \mathrm{~h}$. The translucent ribosomal pellet of ribosomes was then resuspended in $20 \mathrm{mM}$ HEPES-KOH ( $\mathrm{pH} 7.5$ ), $120 \mathrm{mM}$ potassium acetate, $5 \mathrm{mM}$ magnesium acetate, $1 \mathrm{mM}$ DTT and $0.5 \mathrm{mM}$ PMSF.

Ribosome co-sedimentation assay. For the co-sedimentation assay, $25 \mathrm{pmol}$ of ribosomes was incubated with $100 \mathrm{pmol}$ of each protein in the assay buffer containing (25 mM HEPES pH 7.5, $250 \mathrm{mM} \mathrm{KOAc,} 2 \mathrm{mM} \mathrm{Mg}(\mathrm{OAc})_{2}, 0.05 \%$ (v/v) Triton X-100 and $1 \mathrm{mM}$ DTT). The reaction mixture was incubated at $25^{\circ} \mathrm{C}$ for $20 \mathrm{~min}$ and then loaded onto a $0.5 \mathrm{M}$ sucrose cushion prepared in the assay buffer. Ribosomes were pelleted by ultracentrifugation at $267,000 \mathrm{~g}$ at $4{ }^{\circ} \mathrm{C}$ for $1 \mathrm{~h}$ using a TLA100.3 rotor (Beckman). The proteins in the pellet fraction and in the supernatant (following precipitation with trichloroacetic acid) were analysed using SDSPAGE and staining with Coomassie Brilliant Blue.

In vitro translocation assays. Templates for preprolactin ${ }^{65}$, opsin-tagged preprocecropin A, apelin, statherin ${ }^{39}$ and cytochrome $\mathrm{B} 5^{66}$ were generated by $\mathrm{PCR}^{39}$. Transcription reactions were carried out in a volume of $100 \mu \mathrm{l}$ for $2 \mathrm{~h}$ at $37^{\circ} \mathrm{C}$, in the presence of $1 \times$ transcription-optimised buffer (Promega), $10 \mathrm{mM}$ DTT, $0.5 \mu \mathrm{g}$ of template DNA, $0.25 \mathrm{mM}$ each of rATP, rCTP, rGTP and rUTP (Promega), $0.5 \mathrm{mM}$ Cap analogue $\left(\mathrm{m}^{7} \mathrm{G}\left[5^{\prime}\right] \mathrm{ppp}\left[5^{\prime}\right] \mathrm{G}\right.$; New England Biolabs), 80 units of T7 RNA Polymerase (Promega) and 100 units of RNasin Plus RNase Inhibitor (Promega). Transcripts were then purified with an RNeasy Mini kit (Qiagen) according to the manufacturer's instructions.

For post-translational translocation assays, translation reactions $(25 \mu \mathrm{l})$ were carried out using nuclease-treated rabbit reticulocyte lysate (Promega). Translations were performed in the presence of $\left[{ }^{35} \mathrm{~S}\right]$ methionine $(0.769 \mathrm{MBq}$, 43.48 TBq $\mathrm{mmol}^{-1}$; Perkin Elmer). Amino acids minus methionine (Promega) were added to $30 \mu \mathrm{M}$. $1 \mu \mathrm{g}$ of in vitro-transcribed RNA was then added and the sample was incubated for $15 \mathrm{~min}$ at $30^{\circ} \mathrm{C}$. Puromycin was added to $1 \mathrm{mM}$ following the translation and incubated at $30^{\circ} \mathrm{C}$ for a further $5 \mathrm{~min}$ to ensure effective release of the polypeptide from the ribosome. Recombinant SR was added to $10 \mu \mathrm{M}$ as well as 10 eq of RM and the sample was then incubated for $20 \mathrm{~min}$ at $30^{\circ} \mathrm{C}$. Membranes were recovered by centrifugation through an $80 \mu \mathrm{l}$ high-salt cushion $\left(0.75 \mathrm{M}\right.$ sucrose, $0.5 \mathrm{M}$ KOAc, $5 \mathrm{mM} \mathrm{Mg}(\mathrm{OAc})_{2}$, $50 \mathrm{mM}$ HEPES-KOH, pH 7.9) at $100,000 \mathrm{~g}$ for $10 \mathrm{~min}$ at $4^{\circ} \mathrm{C}$ in the TLA 100 rotor (Beckman). The membrane pellet was resuspended in $20 \mu \mathrm{l}$ low-salt buffer, $100 \mathrm{mM}$ sucrose, $100 \mathrm{mM} \mathrm{KOAc}$, $5 \mathrm{mM} \mathrm{Mg}(\mathrm{OAc})_{2}, 50 \mathrm{mM}$ HEPES-KOH pH 7.9, $1 \mathrm{mM}$ DTT and treated with $250 \mu \mathrm{g} \mathrm{ml}^{-1}$ RNase A at $37^{\circ} \mathrm{C}$ for 10 mins. The resulting samples were analysed by SDS-PAGE and phosphorimaging using a Typhoon FLA-7000 (GE Healthcare).

For co-translational reactions, RMs were preincubated with $10 \mu \mathrm{M}$ of the indicated recombinant SR (or an equal volume of SR buffer) on ice for $20 \mathrm{~min}$ and were then recovered by spinning at $100,000 \mathrm{~g}$ for $20 \mathrm{~min}$ and resuspended in RM 
buffer ( $250 \mathrm{mM}$ sucrose, $50 \mathrm{mM}$ HEPES-KOH pH 7.5, $50 \mathrm{mM}$ KOAc, $2 \mathrm{mM}$ $\mathrm{Mg}(\mathrm{OAc})_{2}, 1 \mathrm{mM}$ DTT). Translation reactions $(25 \mu \mathrm{l})$ were carried out as above in the presence of 10 eq of SR/buffer-treated RMs and the samples were incubated for $30 \mathrm{~min}$ at $30^{\circ} \mathrm{C}$. Translated products were then immunoprecipitated by adding 10 volumes of Triton IP buffer ( $10 \mathrm{mM}$ Tris-HCl pH 7.5, $140 \mathrm{mM} \mathrm{NaCl}, 1 \mathrm{mM}$ EDTA, $1 \%(\mathrm{v} / \mathrm{v})$ Triton X-100) and the appropriate antisera (anti-opsin ${ }^{67}$ or anti-pPL (a gift from Sharon Tooze)). The resulting samples were analysed by SDS-PAGE and phosphorimaging using a Typhoon FLA-7000 (GE Healthcare). Data were quantified using Aida (Raytek) and statistical analysis (one-way analysis of variance with Dunnett's post hoc test) was performed using GraphPad (Prism).

Antibodies. The following antibodies, raised in rabbits against the indicated epitopes, were used: Sec61 $\beta$ (PGPTPSGTNC) ${ }^{45}$, SR $\beta$ (CADIQDLEKWLAKIA) ${ }^{64}, \mathrm{SR} \alpha$ (KKFEDSEKAKKPVRC) $^{45}$, Sec62 (a gift from R. Zimmermann-DGETPKSSHEKS) $^{36}$ and Sec62 (Sigma HPA014059-residues 257-395), as well as a mouse monoclonal anti-opsin tag (GPNFYVPFS) antibody ${ }^{67}$. The uncropped immunoblots are provided in Supplementary Fig. 7.

\section{References}

1. Rapoport, T. A. Protein translocation across the eukaryotic endoplasmic reticulum and bacterial plasma membranes. Nature 450, 663-669 (2007).

2. Park, E. \& Rapoport, T. A. Mechanisms of Sec61/SecY-mediated protein translocation across membranes. Ann. Rev. Biophys. 41, 21-40 (2012).

3. Nyathi, Y., Wilkinson, B. M. \& Pool, M. R. Co-translational targeting and translocation of proteins to the endoplasmic reticulum. Biochim. Biophys. Acta 1833, 2392-2402 (2013)

4. Akopian, D., Shen, K., Zhang, X. \& Shan, S. O. Signal recognition particle: an essential protein-targeting machine. Ann. Rev. Biochem. 82, 693-721 (2013).

5. Pool, M. R., Stumm, J., Fulga, T. A., Sinning, I. \& Dobberstein, B. Distinct modes of signal recognition particle interaction with the ribosome. Science 297, 1345-1348 (2002).

6. Halic, M. et al. Following the signal sequence from ribosomal tunnel exit to signal recognition particle. Nature 444, 507-511 (2006).

7. Kurzchalia, T. V. et al. The signal sequence of nascent preprolactin interacts with the $54 \mathrm{~K}$ polypeptide of signal recognition particle. Nature 320, 634-636 (1986).

8. Krieg, U. C., Walter, P. \& Johnson, A. E. Photocrosslinking of the signal sequence of nascent preprolactin to the 54-kilodalton polypeptide of the signal recognition particle. Proc. Natl Acad. Sci. USA 83, 8604-8608 (1986).

9. Siegel, V. \& Walter, P. Elongation arrest is not a prerequisite for secretory protein translocation across the microsomal membrane. J. Cell Biol. 100, 1913-1921 (1985).

10. Gilmore, R., Blobel, G. \& Walter, P. Protein translocation across the endoplasmic reticulum I. Detection in the microsomal membrane of a receptor for the signal recognition particle. J. Cell Biol. 95, 463-469 (1982).

11. Song, W., Raden, D., Mandon, E. C. \& Gilmore, R. Role of Sec61a in the regulated transfer of the ribosome-nascent chain complex from the signal recognition particle to the translocation channel. Cell 100, 333-343 (2000).

12. Becker, T. et al. Structure of monomeric yeast and mammalian Sec61 complexes interacting with the translating ribosome. Science 326, 1369-1373 (2009).

13. Connolly, T., Rapiejko, P. J. \& Gilmore, R. Requirement of GTP hydrolysis for dissociation of the signal recognition particle from its receptor. Science 252, 1171-1173 (1991)

14. Gilmore, R., Walter, P. \& Blobel, G. Protein translocation across the endoplasmic reticulum. II. Isolation and characterization of the signal recognition particle receptor. J. Cell Biol. 95, 470-477 (1982).

15. Miller, J. D., Tajima, S., Lauffer, L. \& Walter, P. The beta subunit of the signal recognition particle receptor is a transmembrane GTPase that anchors the a subunit, a peripheral membrane GTPase, to the endoplasmic reticulum membrane. J. Cell Biol. 128, 273-282 (1995).

16. Bange, G. et al. FlhA provides the adaptor for coordinated delivery of late flagella building blocks to the type III secretion system. Proc. Natl Acad. Sci. USA 107, 11295-11300 (2010).

17. Bange, G. \& Sinning, I. SIMIBI twins in protein targeting and localization. Nat. Struct. Mol. Biol. 20, 776-780 (2013).

18. Peluso, P. et al. Role of the 4.5S RNA in assembly of the bacterial signal recognition particle with its receptor. Science 288, 1640-1643 (2000).

19. Zhang, X., Rashid, R., Wang, K. \& Shan, S. O. Sequential checkpoints govern substrate selection during cotranslational protein targeting. Science 328, 757-760 (2010)

20. Akopian, D., Dalal, K., Shen, K., Duong, F. \& Shan, S. O. SecYEG activates GTPases to drive the completion of cotranslational protein targeting. J. Cell Biol. 200, 397-405 (2013).

21. Schlenker, O., Hendricks, A., Sinning, I. \& Wild, K. The structure of the mammalian signal recognition particle (SRP) receptor as prototype for the interaction of small GTPases with Longin domains. J. Biol. Chem. 281, 8898-8906 (2006).
22. De Franceschi, N. et al. Longin and GAF domains: structural evolution and adaptation to the subcellular trafficking machinery. Traffic 15, 104-121 (2014).

23. Parlitz, R. et al. Escherichia coli signal recognition particle receptor FtsY contains an essential and autonomous membrane-binding amphipathic helix. J. Biol. Chem. 282, 32176-32184 (2007).

24. Stjepanovic, G. et al. Lipids trigger a conformational switch that regulates signal recognition particle (SRP)-mediated protein targeting. J. Biol. Chem. 286, 23489-23497 (2011).

25. Angelini, S., Boy, D., Schiltz, E. \& Koch, H. G. Membrane binding of the bacterial signal recognition particle receptor involves two distinct binding sites. J. Cell Biol. 174, 715-724 (2006)

26. Jadhav, B., Wild, K., Pool, M. R. \& Sinning, I. Structure and Switch Cycle of SRbeta as Ancestral Eukaryotic GTPase Associated with Secretory Membranes. Structure 23, 1838-1847 (2015).

27. Deshaies, R. J. \& Schekman, R. SEC62 encodes a putative membrane protein required for protein translocation into the yeast endoplasmic reticulum. J. Cell Biol. 109, 2653-2644 (1989).

28. Deshaies, R. J., Sanders, S. L., Feldheim, D. A. \& Schekman, R. Assembly of yeast Sec proteins involved in translocation into the endoplasmic reticulum into a membrane-bound multisubunit complex. Nature 349, 806-808 (1991).

29. Chirico, W. J., Waters, M. G. \& Blobel, G. $70 \mathrm{~K}$ heat shock related proteins stimulate protein translocation into microsomes. Nature 332, 805-810 (1988).

30. Deshaies, R. J., Koch, B. D., Werner-Washburne, M., Craig, E. A. \& Schekman, R. A subfamily of stress proteins facilitates translocation of secretory and mitochondrial precursor polypeptides. Nature 332, 800-805 (1988).

31. Matlack, K. E., Misselwitz, B., Plath, K. \& Rapoport, T. A. BiP acts as a molecular ratchet during posttranslational transport of prepro-alpha factor across the ER membrane. Cell 97, 553-564 (1999).

32. Steel, G. J., Fullerton, D. M., Tyson, J. R. \& Stirling, C. J. Coordinated activation of Hsp70 chaperones. Science 303, 98-101 (2004).

33. Ng, D. T., Brown, J. D. \& Walter, P. Signal sequences specify the targeting route to the endoplasmic reticulum membrane. J. Cell Biol. 134, 269-278 (1996).

34. Meyer, H. A. et al. Mammalian Sec61 is associated with Sec62 and Sec63. J. Biol. Chem. 275, 14550-14557 (2000).

35. Tyedmers, J. et al. Homologs of the yeast Sec complex subunits Sec62p and Sec63p are abundant proteins in dog pancreas microsomes. Proc. Natl Acad. Sci. USA 97, 7214-7219 (2000).

36. Noel, P. \& Cartwright, I. L. A Sec62p-related component of the secretory protein translocon from Drosophila displays developmentally complex behavior. EMBO J. 13, 5253-5261 (1994).

37. Lang, S. et al. Different effects of Sec61alpha, Sec62 and Sec63 depletion on transport of polypeptides into the endoplasmic reticulum of mammalian cells J. Cell Sci. 125, 1958-1969 (2012).

38. Lakkaraju, A. K. et al. Efficient secretion of small proteins in mammalian cells relies on Sec62-dependent posttranslational translocation. Mol. Biol. Cell 23, 2712-2722 (2012).

39. Johnson, N. et al. TRC40 can deliver short secretory proteins to the Sec61 translocon. J. Cell Sci. 125, 3612-3620 (2012).

40. Schlenstedt, G., Gudmundsson, G. H., Boman, H. G. \& Zimmermann, R A large presecretory protin translocates both cotranslationally, using signal recognition particle and ribosome, and posttranslationally, without these ribonucleoparticles, when synthesized in the presence of mammalian microsomes. J. Biol. Chem. 265, 13960-13968 (1990).

41. Shao, S. \& Hegde, R. S. A calmodulin-dependent translocation pathway for small secretory proteins. Cell 147, 1576-1588 (2011).

42. Hegde, R. S. \& Keenan, R. J. Tail-anchored membrane protein insertion into the endoplasmic reticulum. Nat. Rev. Mol. Cell Biol. 12, 787-798 (2011).

43. Young, B. P., Craven, R. A., Reid, P. J., Willer, M. \& Stirling, C. J. Sec63p and Kar2p are required for the translocation of SRP-dependent precursors into the yeast endoplasmic reticulum in vivo. EMBO J. 20, 262-271 (2001).

44. Jan, C. H., Williams, C. C. \& Weissman, J. S. Principles of ER cotranslational translocation revealed by proximity-specific ribosome profiling. Science 346, 1257521 (2014)

45. Görlich, D. \& Rapoport, T. A. Protein translocation into proteoliposomes reconstituted from purified components of the endoplasmic reticulum membrane. Cell 75, 615-630 (1993).

46. Kalies, K. U., Rapoport, T. A. \& Hartmann, E. The beta subunit of the Sec61 complex facilitates cotranslational protein transport and interacts with the signal peptidase during translocation. J. Cell Biol. 141, 887-894 (1998).

47. Fulga, T. A., Sinning, I., Dobberstein, B. \& Pool, M. R. SRbeta coordinates signal sequence release from SRP with ribosome binding to the translocon. EMBO J. 20, 2338-2347 (2001).

48. Mandon, E. C., Jiang, Y. \& Gilmore, R. Dual recognition of the ribosome and the signal recognition particle by the SRP receptor during protein targeting to the endoplasmic reticulum. J. Cell Biol. 162, 575-585 (2003). 
49. Johnson, N. et al. The signal sequence influences post-translational ER translocation at distinct stages. PLoS ONE 8, e75394 (2013).

50. Brambillasca, S. et al. Transmembrane topogenesis of a tail-anchored protein is modulated by membrane lipid composition. EMBO J. 24, 2533-2542 (2005).

51. Prinz, A., Behrens, C., Rapoport, T. A., Hartmann, E. \& Kalies, K. U. Evolutionarily conserved binding of ribosomes to the translocation channel via the large ribosomal RNA. EMBO J. 19, 1900-1906 (2000).

52. Harada, Y., Li, H., Wall, J. S., Li, H. \& Lennarz, W. J. Structural studies and the assembly of the heptameric post-translational translocon complex. J. Biol. Chem. 286, 2956-2965 (2011)

53. Voorhees, R. M., Fernandez, I. S., Scheres, S. H. \& Hegde, R. S. Structure of the Mammalian ribosome-sec61 complex to 3.4 a resolution. Cell 157, 1632-1643 (2014).

54. Schaletzky, J. \& Rapoport, T. A. Ribosome binding to and dissociation from translocation sites of the endoplasmic reticulum membrane. Mol. Biol. Cell 17, 3860-3869 (2006)

55. Conti, B. J., Devaraneni, P. K., Yang, Z., David, L. L. \& Skach, W. R. Cotranslational stabilization of Sec62/63 within the ER Sec61 translocon is controlled by distinct substrate-driven translocation events. Mol. Cell 58, 269-283 (2015)

56. Leidig, C. et al. Structural characterization of a eukaryotic chaperone-the ribosome-associated complex. Nat. Struct. Mol. Biol. 20, 23-28 (2013).

57. Seitl, I., Wickles, S., Beckmann, R., Kuhn, A. \& Kiefer, D. The C-terminal regions of YidC from Rhodopirellula baltica and Oceanicaulis alexandrii bind to ribosomes and partially substitute for SRP receptor function in Escherichia coli. Mol. Microbiol. 91, 408-421 (2014).

58. Muller, L. et al. Evolutionary gain of function for the ER membrane protein Sec62 from yeast to humans. Mol. Biol. Cell 21, 691-703 (2010).

59. Webb, G. C., Akbar, M. S., Zhao, C. \& Steiner, D. F. Expression profiling of pancreatic beta cells: glucose regulation of secretory and metabolic pathway genes. Proc. Natl Acad. Sci. USA 97, 5773-5778 (2000).

60. Bogomolovas, J., Simon, B., Sattler, M. \& Stier, G. Screening of fusion partners for high yield expression and purification of bioactive viscotoxins. Protein Expr. Purif. 64, 16-23 (2009).

61. Studier, F. W. Protein production by auto-induction in high density shaking cultures. Protein Expr. Purif. 41, 207-234 (2005)

62. Walter, P. \& Blobel, G. Preparation of microsomal membranes for cotranslational protein translocation. Meth. Enz. 96, 84-93 (1983).

63. Martoglio, B., Hauser, S. \& Dobberstein, B. in Cell Biology: A Laboratory Handbook 265-273 (Academic Press, 1997).

64. Bacher, G., Pool, M. \& Dobberstein, B. The ribosome regulates the GTPase of the $\beta$-subunit of the signal recognition particle receptor. J. Cell Biol. 146, 723-730 (1999).
65. High, S. et al. Site-specific photocross-linking reveals that Sec61p and TRAM contact different regions of a membrane inserted signal sequence. J. Biol. Chem. 268, 26745-26751 (1993).

66. Rabu, C., Wipf, P., Brodsky, J. L. \& High, S. A precursor-specific role for $\mathrm{Hsp} 40 / \mathrm{Hsc} 70$ during tail-anchored protein integration at the endoplasmic reticulum. J. Biol. Chem. 283, 27504-27513 (2008).

67. Adamus, G., Arendt, A. \& Hargrave, P. A. Genetic control of antibody response to bovine rhodopsin in mice: epitope mapping of rhodopsin structure. J. Neuroimmunol. 34, 89-97 (1991).

\section{Acknowledgements}

We thank Richard Zimmermann, Sharon Tooze and Bernhard Dobberstein for reagents B.J. was funded by Graduiertenkolleg GRK1188 from the German Research Council (DFG). M.M. and N.J. were funded by the BBSRC doctoral training grants (BB/J014478/1 and $\mathrm{BB} / \mathrm{F} 017227 / 1$, respectively). I.S. is an investigator of the cluster of excellence: CellNetworks and acknowledges support by the DFG (SFB638).

\section{Author contributions}

B.J. performed and helped in designing the experiments. N.J. and M.M. performed and helped in designing the in vitro translocation experiments. S.H. designed the experiments, analysed the data and edited the paper. M.R.P. and I.S. conceived the project, designed the experiments, analysed the data and wrote the manuscript. M.R.P. also performed experiments.

\section{Additional information}

Supplementary Information accompanies this paper at http://www.nature.com/ naturecommunications

Competing financial interests: The authors declare no competing financial interests.

Reprints and permission information is available online at http://npg.nature.com/ reprintsandpermissions/

How to cite this article: Jadhav, B. et al. Mammalian SRP receptor switches the Sec61 translocase from Sec62 to SRP-dependent translocation. Nat. Commun. 6:10133 doi: 10.1038/ncomms10133 (2015)

(c) (i) This work is licensed under a Creative Commons Attribution 4.0 International License. The images or other third party material in this article are included in the article's Creative Commons license, unless indicated otherwise in the credit line; if the material is not included under the Creative Commons license, users will need to obtain permission from the license holder to reproduce the material To view a copy of this license, visit http://creativecommons.org/licenses/by/4.0/ 